\title{
Ausgewählte methodische Aspekte bei der Nutzung von Qualitätsindikatoren auf der Basis von Routinedaten
}

\section{Kontext und Problemstellung \\ $\nabla$}

Qualitätsindikatoren haben in Deutschland in den letzten Jahren zunehmend mehr Aufmerksamkeit erhalten. Im stationären Sektor wurde die Auseinandersetzung mit Indikatoren vor allem durch die gesetzliche Verpflichtung zur externen Qualitätssicherung (BQS-Verfahren) angestoßen. Im ambulanten Sektor bestehen seit rund 10 Jahren einzelne Initiativen. So werden z.B. in hausärztlichen Leitlinien der Leitliniengruppe Hessen Indikatoren zur Darstellung der Leitliniennähe auf der Basis von Verordnungsdaten vorgeschlagen (face und content validity) und in Feedbackberichten für Ärzte über ihr Verordnungsverhalten (z.B. im Rahmen von Qualitätszirkeln) ausgewiesen (http://www.leitlinien.de). Auch Arztnetze nutzen Indikatoren als Steuerungsinformation [1]. Die Interpretation erfolgte hierbei bislang durch die Ärzte selbst (Überprüfung des Erreichens selbstgesetzter Ziele) unter Berücksichtigung der Grenzen des Indikators. Gegenwärtig bestehen Aktivitäten zur Testung von Indikatoren, die längerfristig für eine qualitätsorientierte Vergütung im ambulanten Sektor herangezogen werden können (AQIK).

Inzwischen haben sich Standards zur Entwicklung von Indikatoren herausgebildet (z.B. RANDVerfahren) und mit dem Qualify-Instrument sind „Qualitätsanforderungen“ - methodische Gütekriterien - hinsichtlich Relevanz, Wissenschaftlichkeit und Praktikabilität benannt (http:// www.bqs-qualitätsindikatoren.de/qualify).

Wichtige Aspekte stellen neben der Problematik der Risikoadjustierung (s. Beitrag von Heller) die Überprüfung der Datenqualität (Einfluss auf Zähler und Nenner) und die Ermittlung eines belastbaren Referenzbereiches dar.

\section{Methodische Anforderungen \\ $\nabla$}

a) Qualität von Routinedaten: Routinedaten zeichnen sich durch Vollständigkeit, fehlenden Recall- und fehlenden Selektionsbias aus. Therapiespezifische Indikatoren erfordern sowohl eine Darlegung des Algorithmus zur Definition von Versicherten mit der Zielerkrankungen (interne Validierung der Diagnosen) als auch eine explizite Beschreibung von Zähler und Nenner (z.B. welche Personengruppe stellt den Nenner dar?) [2]. Eine Überprüfung der Belastbarkeit der herangezogenen Routinedaten und der gewählten Algorithmen kann durch einen Vergleich mit ei- ner anderen Datengrundlage und Methodik (Primärerhebung in Praxen, Auswahl der Patienten mit Zieldiagnose durch den Arzt) erfolgen. So zeigte sich z.B. bei fünf von sieben auf der Basis von Routinedaten zur Therapie von Herzkreislauferkrankungen erhobenen PMV-Zirkelindikatoren im Vergleich zu einer Primärerhebung [3] eine sehr gute Übereinstimmung.

b) Adjustierung des Zielbereiches: $\mathrm{Zu}$ einem Qualitätsindikator gehört ein Referenzbereich. Bislang erfolgt diese Festlegung meistens mittels einer Referenzgruppe (z.B. 10\% der besten Praxen, Leitliniengruppe) oder durch Expertenkonsens. Zur Ermittlung eines Referenzbereiches ist es erforderlich, den Anteil der Patienten zu kennen, bei denen begründet das zu untersuchende Qualitätsmerkmal nicht zur Anwendung kommt. Hierzu sind Primärerhebungen notwendig, da bestimmte ärztliche Entscheidungen (z. B. bettlägeriger Patient mit schlechter Prognose; Probleme im Zusammenhang mit Multimedikation, Patientenpräferenzen) anhand der Routinedaten nicht abgebildet und somit auch nicht in die Definition des Nenners einbezogen werden können.

\section{Schlussfolgerung}

$\checkmark$

Die Implementierung von Qualitätsindikatoren und ihrer Zielbereiche für den ambulanten Sektor erfordert noch methodische Entwicklungsarbeit. Routinedaten werden eine relevante Datenquelle darstellen.

Autorenerklärung: Die Durchführung der Pharmakotherapiezirkel wurde durch den VdAK/AEV und die KV Hessen finanziert, die Bundesärztekammer fördert das Projekt zur Leitliniencompliance.

\section{Literatur}

1 Stock J, Szecsenyi J. Stichwort Qualitätsindikatoren. Erste Erfahrungen in der Praxis. Bonn, Frankfurt/M: KomPart-Verlagsgesellschaft, 2007

2 Schubert I, Ihle P, Köster I. Verwendung von GKV-Diagnosen in der Sekundärdatenforschung.In: Swart E, Ihle P, Hrsg (Hrsg). Routinedaten im Gesundheitswesen. Handbuch Sekundärdatenanalyse: Grundlagen, Methoden und Perspektiven. Bern: Hans Huber, 2005: 235-41

3 Karbach U, Hagemeister J, Pfaff H, Schubert I, Höpp HW. Messbarkeit der de-facto Compliance kardiovaskulärer Leitlinien und ihrer Determinanten. Abschlussbericht in Vorbereitung. Posterabstract zum 6. Deutschen Kongress für Versorgungsforschung 2007. Prävention und Gesundheitsförderung 2007; Suppl 1: 112-113
I. Schubert

Qualitätsmanagement

Schlüsselwörter

Qualitätsindikatoren

ambulante Versorgung

Routinedaten

evidenzbasierte Leitlinien

Validierung

Key words

quality indicators

- ambulatory care

administrative data

- evidence based guidelines

validity assessment

Institut

PMV forschungsgruppe an der Klinik und Poliklinik für Psychiatrie und Psychotherapie des Kindes- und Jugendalters der Universität zu Köln

Bibliografie

DOI 10.1055/s-0028-1085599

Dtsch Med Wochenschr 2008;

133: S155 - (c) Georg Thieme

Verlag KG Stuttgart - New York . ISSN 0012-0472

Korrespondenz

Dr. Ingrid Schubert

PMV forschungsgruppe an der Klinik und Poliklinik für Psychiatrie und Psychotherapie des Kindes- und Jugendalters der Universität zu Köln

Herderstraße 52-54 50931 Köln

Tel. +49 (221) 478-6545 eMail

ingrid.schubert@uk-koeln.de 